\title{
Azithromycin treatment modifies airway and blood gene expression networks in neutrophilic COPD
}

\author{
Katherine J. Baines ${ }^{1}$, Thomas K. Wright ${ }^{1}$, Peter G. Gibson ${ }^{1,2}$, Heather Powell ${ }^{2}$, \\ Philip M. Hansbro' and Jodie L. Simpson ${ }^{1}$
}

Affiliations: ${ }^{1}$ Priority Research Centre for Healthy Lungs and Hunter Medical Research Institute, Faculty of Health and Medicine, The University of Newcastle, Callaghan, Australia. ${ }^{2}$ Dept of Respiratory and Sleep Medicine, Hunter New England Area Health Service, Newcastle, Australia.

Correspondence: Katherine J. Baines, Level 2 West, Hunter Medical Research Institute, Lot 1, Kookaburra Circuit, New Lambton Heights, NSW 2310, Australia. E-mail: katherine.baines@newcastle.edu.au

ABSTRACT Long-term, low-dose azithromycin reduces exacerbation frequency in chronic obstructive pulmonary disease (COPD), yet the mechanism remains unclear. This study characterised genome-wide gene expression changes in patients with neutrophilic COPD following long-term, low-dose azithromycin treatment.

Patients with neutrophilic COPD $\left(>61 \%\right.$ or $>162 \times 10^{4}$ cells per $\mathrm{mL}$ sputum neutrophils) were randomised to receive either azithromycin or placebo for 12 weeks. Sputum and blood were obtained before and after 12 weeks of treatment. Gene expression was defined using microarrays. Networks were analysed using the Search Tool for the Retrieval of Interacting Gene database.

In sputum, 403 genes were differentially expressed following azithromycin treatment (171 downregulated and 232 upregulated), and three following placebo treatment (one downregulated and two upregulated) compared to baseline (adjusted $\mathrm{p}<0.05$ by paired t-test, fold-change $>1.5$ ). In blood, 138 genes were differentially expressed with azithromycin (121 downregulated and 17 upregulated), and zero with placebo compared to baseline (adjusted $\mathrm{p}<0.05$ by paired t-test, fold-change $>1.3$ ). Network analysis revealed one key network in both sputum (14 genes) and blood (46 genes), involving interferon-stimulated genes, human leukocyte antigens and genes regulating $\mathrm{T}$-cell responses.

Long-term, low-dose azithromycin is associated with downregulation of genes regulating antigen presentation, interferon and T-cell responses, and numerous inflammatory pathways in the airways and blood of neutrophilic COPD patients.

@ERSpublications

Azithromycin modifies gene expression in COPD http://ow.ly/Mqpv30lLXUM

Cite this article as: Baines KJ, Wright TK, Gibson PG, et al. Azithromycin treatment modifies airway and blood gene expression networks in neutrophilic COPD. ERJ Open Res 2018; 4: 000312018 [https://doi.org/10.1183/23120541.00031-2018].

This article has supplementary material available from openres.ersjournals.com

This study is registered at www.anzctr.org.au with identifier number ACTRN 12609000259246. Data sharing is not in place for this trial.

Received: April 232018 | Accepted after revision: Sept 072018

Copyright $\odot$ ERS 2018. This article is open access and distributed under the terms of the Creative Commons Attribution Non-Commercial Licence 4.0. 


\section{Introduction}

Chronic obstructive pulmonary disease (COPD) is a common illness that poses a major global health burden $[1,2]$. It is characterised by airway neutrophilia, persistent airflow obstruction and exacerbations. The prevention of exacerbations in COPD is of great importance given their association with lung function decline, impairment of quality of life, and mortality risk [3-5].

Azithromycin is a macrolide antibiotic used in the treatment of acute, infectious exacerbations of COPD. Long-term use of low-dose azithromycin has been shown to reduce the number of exacerbations in a range of respiratory diseases, including asthma [6], COPD [7-9], cystic fibrosis [10, 11] and non-cystic fibrosis bronchiectasis [12, 13]. Additional benefits include improved lung function in diffuse panbronchiolitis [14] and bronchiolitis obliterans syndrome [15], and a reduced rate of lung function decline in cystic fibrosis [11]. While such clinical outcomes are promising for the future management of these diseases, the underlying mechanism remains unclear.

Macrolides, in addition to their antimicrobial properties, have numerous anti-inflammatory and immunomodulatory effects. Using microarrays to define how macrolides affect gene expression across the whole genome will facilitate the understanding of how they impact airway inflammation, and potentially identify targets for future drug development that do not have the same issues with antibiotic resistance [16]

We hypothesised that 12 weeks of azithromycin treatment would modify gene expression profiles in the airway and blood of patients with neutrophilic COPD. This study characterised these gene expression changes using microarrays to identify the underlying molecular mechanisms responsible for the clinical effects of long-term, low-dose azithromycin in COPD.

\section{Materials and methods}

\section{Study design and population}

This randomised controlled trial was conducted between April 2009 and December 2011, and clinical and inflammatory results have been published previously [8]. Recruitment for this study targeted adults with doctor diagnosed symptomatic COPD, as described in detail previously [8], with stable persistent neutrophilic inflammation, defined by a sputum differential cell count demonstrating $>61 \%$ or $>162 \times 10^{4}$ cells per $\mathrm{mL}$ neutrophils on two separate occasions (with at least one being the screening visit). These cut-offs, as well as inclusion and exclusion criteria have been discussed previously [8].

Eligible participants $(n=30)$ were randomly allocated $(1: 1)$ to receive oral azithromycin $250 \mathrm{mg}$ daily or placebo for 12 weeks. In addition to the screening visit, participants attended four visits at monthly intervals with the final study visit conducted 4 weeks after the end of treatment.

\section{Ethics statement}

Participants gave written informed consent. The Hunter New England Area Health Service and University of Newcastle Research Human Ethics Committees approved the study (06/12/13/3.08 and H-2008-0272) and it was registered with the Australian New Zealand Clinical Trials Registry ACTRN 12609000259246.

\section{Study protocol}

At screening (visit 1) demographics, pre- and post-bronchodilator spirometry, skin-prick testing, medication history, smoking status, and exhaled carbon monoxide were assessed.

At visit 2 (baseline randomisation) mucus hypersecretion, St George's Respiratory Questionnaire [17], symptom visual analogue scores, Clinical COPD Questionnaire [18], and modified Medical Research Council dyspnoea scale were performed. Induced sputum and peripheral blood (PAXgene Blood RNA tubes; PreAnalytix, Hombrechtikon, Switzerland) were collected at the baseline randomisation visit and after 12-weeks of azithromycin or placebo treatment, for gene expression profiling.

\section{Sputum induction and analysis}

Sputum was induced at baseline and after the 12-week treatment period with hypertonic saline (4.5\%), as previously described [19]. Selected sputum was dispersed using dithiothreitol for differential cell counts or stored by placing directly into RLT buffer for RNA extraction. Total cell counts of leukocytes and their viability were performed on filtered suspensions. Cytospins were prepared, stained (May-Grunwald Geimsa) and a differential cell count obtained from 400 non-squamous cells. Following centrifugation, supernatant was stored at $-80^{\circ} \mathrm{C}$.

\section{Whole-genome gene expression microarrays}

RNA was extracted from induced sputum using RNeasy Mini Kits (Qiagen, Hilden, Germany), whereas peripheral blood collected in PAXgene RNA tubes was extracted using the PAXgene Blood RNA Kit 
(Qiagen) according to the manufacturers' instructions. RNA was quantitated using Quant-iT RiboGreen RNA Quantitation Assay Kits (Molecular Probes Inc, Invitrogen, Eugene, OR, USA). A total of $500 \mathrm{ng}$ RNA was reverse-transcribed into cRNA and biotin-uridine-5'-triphosphate labelled using Illumina TotalPrep RNA Amplification Kits (Ambion, Austin, TX, USA). A total of 750 ng cRNA was hybridised to Illumina Sentrix HumanRef-8 Version 2 Expression BeadChips and scanned using an Illumina Bead Station and captured using BeadScan 3.5.11 (Illumina, San Diego, CA, USA). Samples and gene profiling results were included in the analysis if the sample was of suitable purity (optical density $260 \mathrm{~nm} / 280 \mathrm{~nm}$ : 1.7-2.1) and was successfully amplified (sufficient cRNA generated) and hybridised (95th percentile of fluorescence score $>500$ ) and the data passed quality controls in GeneSpring GX (correlation coefficients and principle component analysis plots; Agilent Technologies, Santa Clara, CA, USA).

The data discussed in this publication have been deposited in NCBI's Gene Expression Omnibus [20] and are accessible through GEO Series accession number GSE119314 (www.ncbi.nlm.nih.gov/geo/query/acc. cgi?acc $=$ GSE119314).

\section{Network analysis}

We used the Search Tool for the Retrieval of Interacting Genes (STRING) v9.1 (http://string-db.org/) to identify gene networks amongst our differentially expressed genes. STRING is an online protein-protein interaction database curated from the literature that predicts associations from systemic genome comparisons. All network diagrams presented here are based on high confidence scores $(>0.7)$, to retain the highest possible stringency, as well as at least one network consisting of a node with more than three neighbours.

\section{Statistical methods}

Statistical analysis was performed using Stata 11 (StataCorp, College Station, TX, USA). Results are presented as mean \pm SD or median (interquartile range) with t-tests for parametric data and Wilcoxon rank sum tests for nonparametric data. Paired data were analysed using the Wilcoxon signed rank test. Categorical data were compared using the Chi-squared or Fisher's exact test as appropriate. p-values $<0.05$ were considered significant.

Microarray data were exported using Genome Studio (Illumina) and analysed using GeneSpring GX12.5 (Agilent Technologies). Data were log transformed and quantile normalisation performed. Data were filtered on expression level, and only genes with a raw fluorescence of $>250$ in all samples were included in further analysis. Hierarchical clustering analysis was performed using the Euclidean algorithm with Ward's linkage. The Euclidian algorithm takes the standard sum of squared distance between two entities. Ward's linkage, based on the ANOVA approach, computes the sum of squared errors around the mean for each cluster, then the two clusters are joined to minimise the increase in error. Differential gene expression before and after azithromycin treatment or placebo was determined using paired t-tests, and further filtered on a volcano plot, whereby those genes with adjusted $\mathrm{p}<0.05$ (Benjamini-Hochberg) and fold-change $>1.5$ (sputum) or $>1.3$ (blood) were included. Gene ontology categories and pathways that were overrepresented in differentially expressed gene lists were determined by using GeneSpring GX12.5 where an adjusted $\mathrm{p}<0.05$ was considered significant.

\section{Results}

\section{Sample details}

All clinical and inflammatory data has been published previously [8]. Briefly, the mean $\pm \mathrm{SD}$ age was $70 \pm 7.6$ years, $63.3 \%$ were female, and $73.3 \%$ were ex-smokers with a mean \pm SD smoking history of $46.11 \pm 36.11$ pack-years. The mean \pm SD forced expiratory volume in $1 \mathrm{~s}$ (FEV1) was $53.69 \pm 13.74 \%$ predicted and the mean $\pm \mathrm{SD} \mathrm{FEV}_{1} / \mathrm{FVC}$ was $57.79 \pm 11.24$. There were no significant differences in any clinical or inflammatory parameters between the placebo and azithromycin groups.

Microarray analysis was performed on blood ( $\mathrm{n}=11$ azithromycin group; $\mathrm{n}=12$ placebo group) and sputum ( $\mathrm{n}=11$ azithromycin group; $\mathrm{n}=13$ placebo group) in a subset of patients who had paired samples from baseline and post-treatment visits, as well as sufficient RNA available. Regarding blood samples, five patients did not have paired samples, and a further two did not have sufficient RNA available for analysis. Regarding sputum samples, three patients did not have paired samples, and a further three did not have sufficient RNA available for analysis.

Exacerbation data has been published in detail previously [8]. Of the subset from the original study that were analysed, six (46.2\%) out of 13 in the placebo group and three (21.4\%) out of 14 in the azithromycin group experienced an exacerbation. The median (quartile 1, quartile 3) (range) number of exacerbations experienced were $0(0,1)(0-2)$ and $0(0,0)(0-1)$ for the placebo and azithromycin groups, respectively. 
Differential gene expression in sputum after azithromycin or placebo treatment

Following azithromycin treatment, the expression of 1877 cRNAs was significantly altered $(\mathrm{p}<0.05$ paired t-test) compared to baseline. Further filtering on a volcano plot (adjusted $\mathrm{p}<0.05$ paired $\mathrm{t}$-test, fold-change $>1.5$ ) resulted in 667 cRNAs that were differentially expressed. This corresponded to 403 genes that were differentially expressed (with unannotated, predicted targets and duplicated probes removed) (171 downregulated, 232 upregulated). Gene ontologies for biological processes that were significantly altered with azithromycin treatment were identified (table 1). Of interest, there were 35 differentially expressed genes involved in immune system processes and nine genes involved in antigen processing and presentation (all genes involved in antigen presentation were also involved in immune system processes) (table 2). Gene expression patterns are detailed in figure 1.

Following treatment with placebo, the expression of 227 cRNAs was significantly altered $(\mathrm{p}<0.05$ paired t-test) compared to baseline. Further filtering on a volcano plot (adjusted $\mathrm{p}<0.05$ paired t-test, fold-change $>1.5$ fold) resulted in only three genes being differentially expressed. These were RASA4P (RAS p21 protein activator 4, non-coding RNA, 1.56-fold upregulated), ATP6V0D1 (ATPase, $\mathrm{H}+$ transporting, lysosomal $38 \mathrm{kDa}$, V0 subunit d1 mRNA, 1.56-fold downregulated) and HLA-H (Major histocompatibility complex, class I, H (pseudogene), non-coding RNA, 1.62-fold upregulated). As the expression of these three genes were significantly altered following treatment with both azithromycin and placebo, the altered regulation could not be confidently attributed to azithromycin and they were removed from further analysis.

\section{Differential gene expression in peripheral blood after azithromycin or placebo treatment}

Following azithromycin treatment, the expression of 2398 cRNAs was altered $(\mathrm{p}<0.05$ paired $\mathrm{t}$-test) compared to baseline. Further filtering using a volcano plot (adjusted $\mathrm{p}<0.05$, fold-change $>1.5$ ) resulted in 25 cRNAs that were differentially expressed. This corresponded to 22 differentially expressed genes (with unannotated, predicted targets and duplicated probes removed), far fewer than the number seen in sputum. All genes were downregulated (supplementary table S1). There were no significant gene ontologies due to the small number of differentially regulated genes. The use of an identical fold-change cut-off in blood and sputum was, however, deemed impractical as the concentration of azithromycin is often 10-fold lower in plasma than in tissues due to its high cellular penetration [21]. Consequently, the fold-change cut-off was lowered to 1.3 to more accurately reflect this difference, resulting in 166 significantly altered cRNAs (138 genes). 48 of these genes were involved in immune system processes (table 3 ). Figure 2 shows the shift in gene expression profile after azithromycin treatment.

Following treatment with placebo, the expression of 1374 cRNAs was altered $(\mathrm{p}<0.05$ paired $\mathrm{t}$-test) compared to baseline. However, none of these genes reached significance on filtering using the volcano plot (adjusted $\mathrm{p}<0.05$ paired t-test, fold-change $>1.3$ ).

Network analysis of differentially expressed genes in sputum and blood following azithromycin To investigate potential pathways underlying the differential gene expression the STRING database was used. From the 35 genes that were differentially expressed in sputum with a fold-change of $>1.5$ and involved in immune system processes and antigen processing and presentation, 33 genes were recognised by the STRING database. One key network was found that involved 14 genes (figure 3). Key nodes were

TABLE 1 Gene ontology $(G O)$ biological processes altered in the airways with azithromycin treatment

$\begin{array}{lcc}\text { GO biological process } & \begin{array}{c}\text { Number of differentially } \\ \text { expressed genes }\end{array} & \begin{array}{c}\text { Adjusted p-value } \\ \text { Translational elongation }\end{array} \\ \text { Translation } & 28 & 1.25 \times 10^{-17} \\ \text { Immune response } & 39 & 7.99 \times 10^{-18} \\ \text { Energy coupled proton transmembrane transport, } & 31 & 0.004 \\ \quad \text { against electrochemical gradient } & 5 & 0.012 \\ \text { Cellular macromolecule biosynthetic process } & 74 & 0.016 \\ \text { Immune system processes } & 36 & 0.017 \\ \text { Macromolecule biosynthetic process } & 74 & 0.017 \\ \text { Antigen processing and presentation } & 9 & 0.017 \\ \text { Actin filament-based process } & 15 & 0.035 \\ \text { Antigen processing and presentation of peptide antigen } & 6 & 0.042 \\ \text { Cellular biosynthetic process } & 85 & 0.044\end{array}$


TABLE 2 Differentially expressed genes in sputum after azithromycin treatment involved in immune system processes

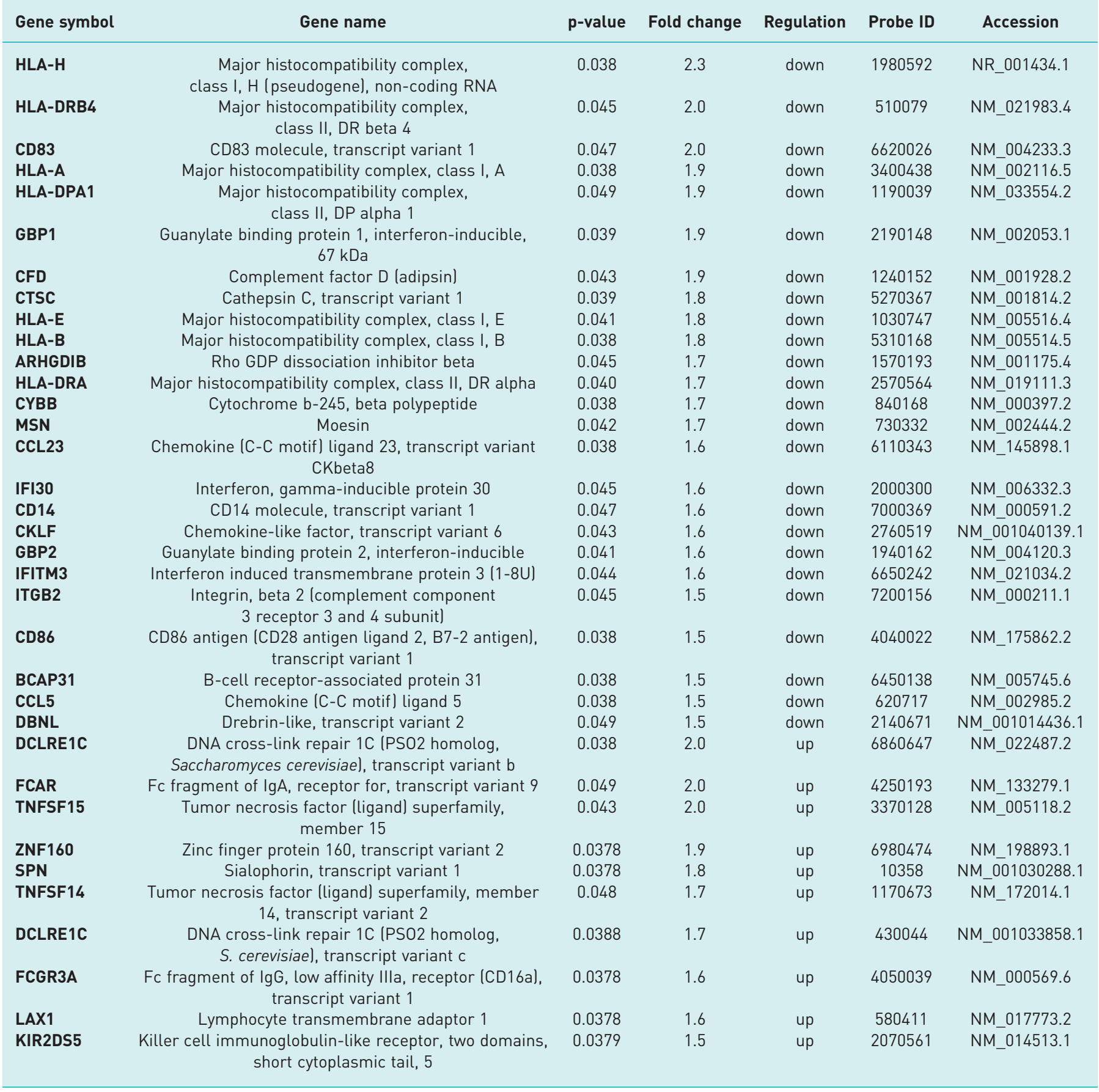

determined by the number of connections with other differentially expressed genes. Those with $\geqslant 3$ connections are shown in supplementary table S2.

From the 138 genes that were differentially expressed in blood with a fold-change of $>1.3,135$ were recognised by the STRING database. One key network was found that involved 46 genes (figure 4). Key nodes were determined by the number of connections with other differentially expressed genes. Those with $\geqslant 3$ connections are shown in supplementary table S3.

\section{Discussion}

This study examined the underlying mechanisms of long-term, low-dose azithromycin in neutrophilic COPD. Patients with stable, neutrophilic COPD were administered either low-dose azithromycin or placebo 


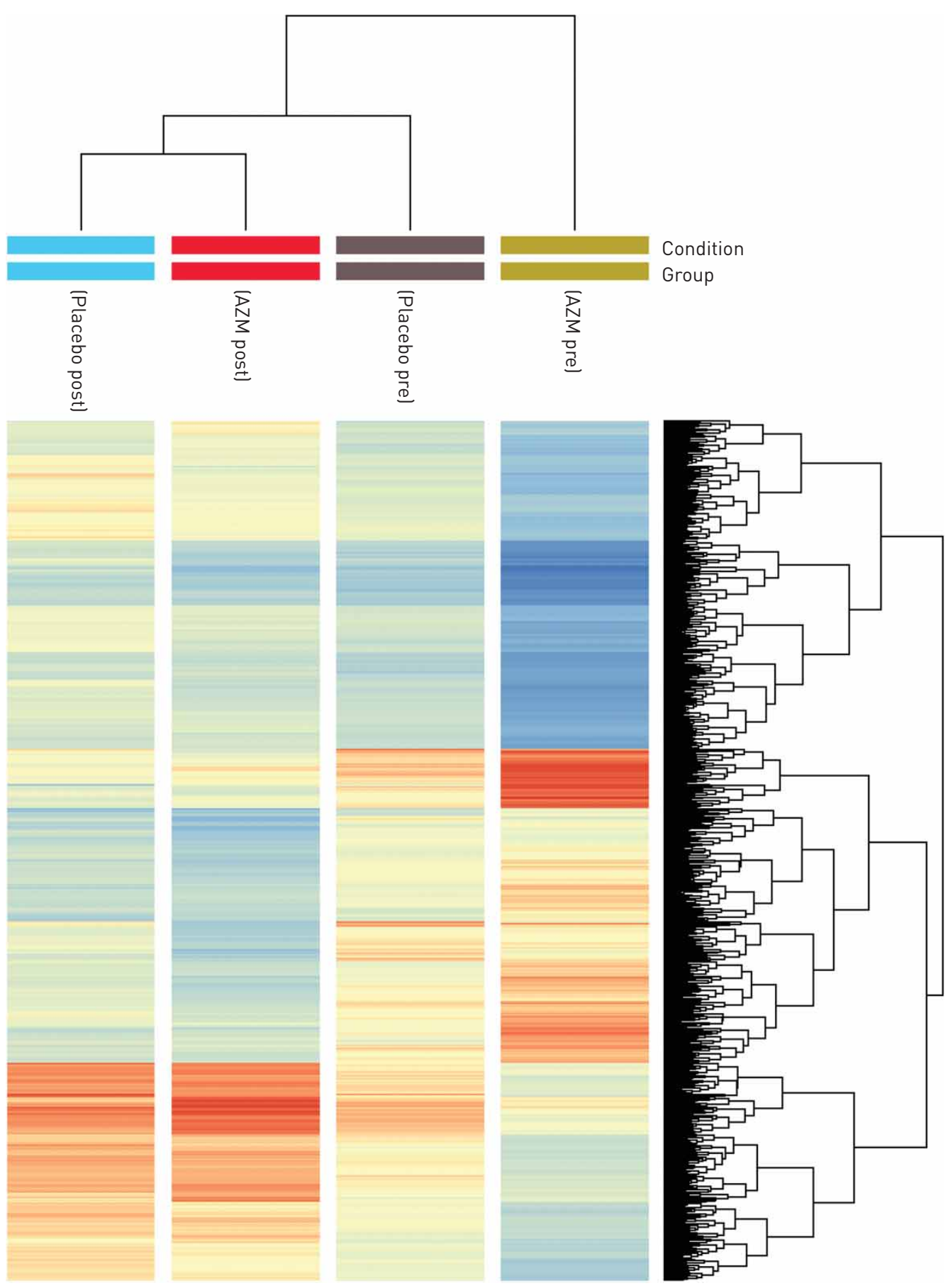

FIGURE 1 Microarray clustering of differentially expressed genes in sputum. Genes are clustered in rows, with red indicating high expression, yellow intermediate expression and blue low expression. Treatment groups are clustered in columns. Groups are clustered to reflect similarities and differences in gene expression (as per the Euclidean algorithm with Ward's linkage). AZM: azithromycin.

over 12 weeks. Treatment with azithromycin resulted in significant changes in gene profiles in sputum and blood, including the downregulation of genes associated with antigen presentation, inflammation, and interferon and T-cell responses. Gene profiles showed only minor changes following treatment with placebo.

COPD exacerbations can be triggered by infectious and non-infectious agents, and the mechanism by which azithromycin prevents exacerbations is probably complex and multi-factorial. Previous research has demonstrated that long-term, low-dose azithromycin suppresses pro-inflammatory cytokine production, enhances macrophage phagocytosis $[22,23]$ and the expression of anti-inflammatory cytokines [24], and 
TABLE 3 Differentially expressed genes in blood after azithromycin treatment involved in immune system processes

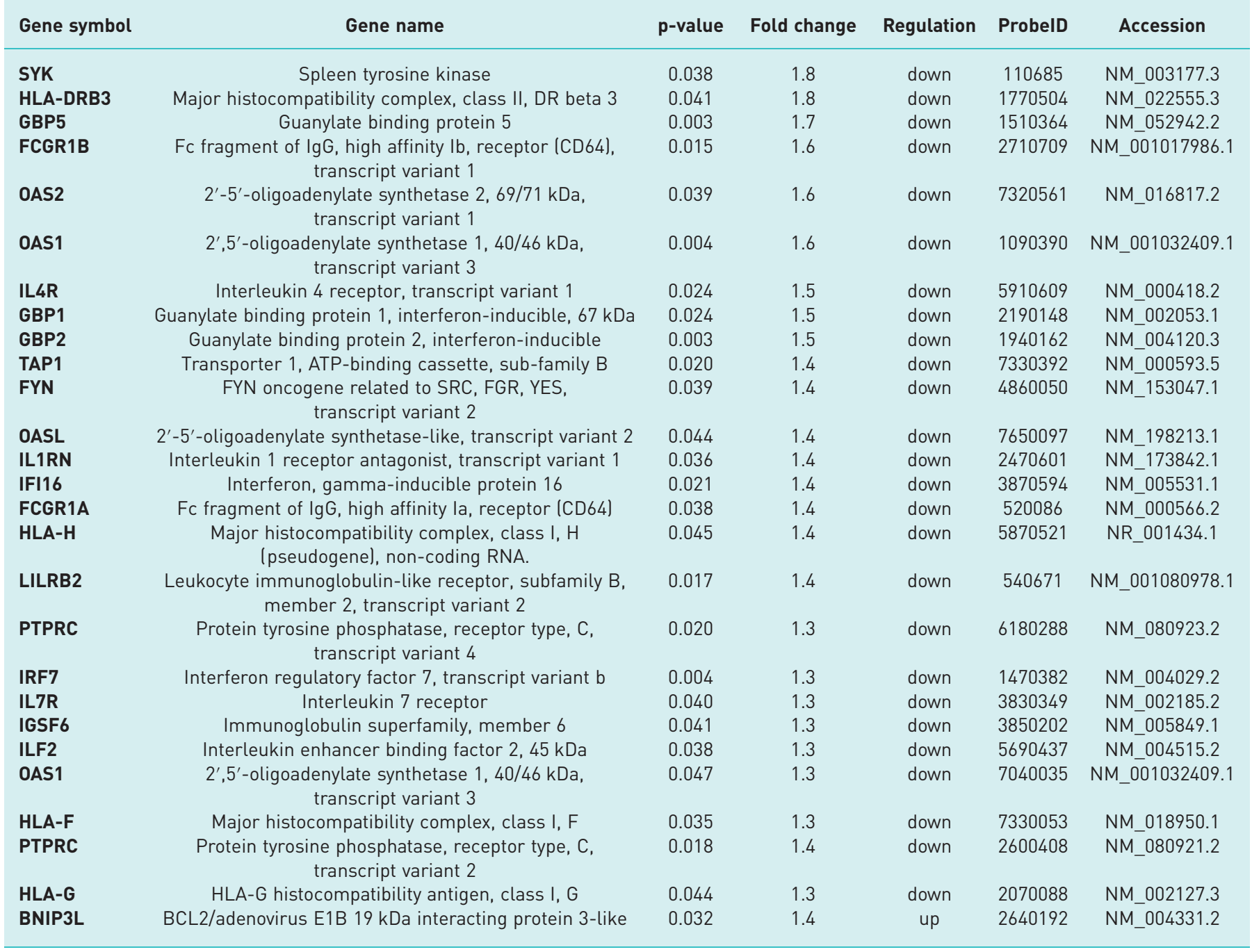

modulates interferon [25-29] and T-cell responses [30, 31]. Importantly, these functions are not universal to all macrolides (reviewed in [32]), with slight structural differences affecting antimicrobial and immunomodulatory properties [28].

Our research demonstrated that azithromycin was associated with a downregulation of several human leukocyte antigen (HLA) molecules in the airways (HLA-A, HLA-B, HLA-DPA1, HLA-DRA and HLA-DRB4). HLA-A and HLA-B are subtypes of major histocompatibility complex (MHC) class I surface receptors, while HLA-DPA1, HLA-DRA and HLA-DRB4 are subtypes of MHC class II surface receptors. MHC class I and II receptors are involved in the presentation of antigens to $\mathrm{CD}^{+}$and $\mathrm{CD} 8^{+} \mathrm{T}$-cells, respectively. TAP-1 expression, which is essential for the formation of MHC-I-peptide complexes (reviewed in [33]) and therefore, for effective antigen presentation to $\mathrm{CD}^{+} \mathrm{T}$-cells [34], was also downregulated following treatment with azithromycin. Antigen presentation is a critical step in T-cell activation, and therefore for the initiation of the adaptive immune responses in general. Consequently, azithromycin may suppress inflammatory responses in the airways by impairing antigen presentation and therefore limiting the immune system's capacity to identify and respond to antigen. The mechanism, however, is unclear.

Azithromycin was also associated with the downregulation of several interferon-stimulated genes in blood (EIF2AK2, EPSTI, GBP1, GBP2, GBP5, HERC5, IFI16, IFIT1, MX1, OAS1, OAS2, OASL and UBE2L6) and sputum (GBP1, GBP2, IFI30 and IFITM3). This may be explained by the downregulation of several genes that are involved in the induction of interferon expression (TRIM25 and IRF7) and interferon signalling (STAT1 and IRF9). TRIM25 regulates the polyubiquitination of RIG-I, a pattern recognition receptor that responds to viral dsRNA. This process results in the induction of a signalling cascade, which leads to the 


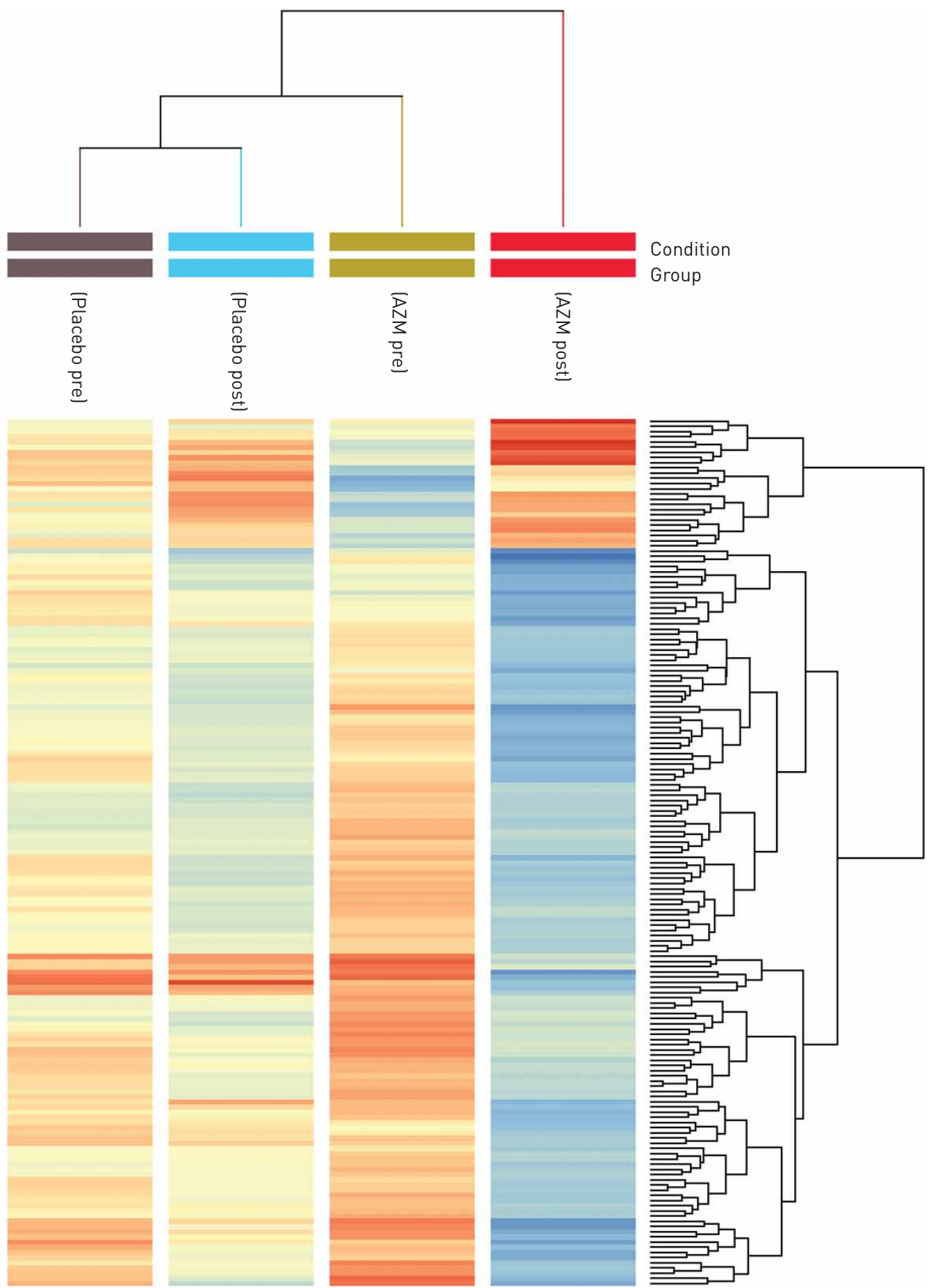

FIGURE 2 Microarray clustering of differentially expressed genes in blood. Genes are clustered in rows, with red indicating high expression, yellow intermediate expression and blue low expression. Treatment groups are clustered in columns. Groups are clustered to reflect similarities and differences in gene expression las per the Euclidean algorithm with Ward's linkage). AZM: azithromycin.

activation of IRF-7, a transcription factor that induces the expression of type-I interferons (IFN- $\alpha$ and IFN- $\beta$ ). Once interferons are expressed, they are released from the cell and bind to interferon receptors on the surface of neighbouring cells. Interferon receptor activation induces a signalling cascade that culminates in the expression of interferon-stimulated genes. Two key transcription factors involved in this signalling cascade, STAT1 and IRF9, were downregulated following azithromycin treatment. STAT1 forms a STAT1-STAT1 homodimer to induce the expression of IFN- $\gamma$-associated genes, and a STAT1-STAT2 heterodimer that complexes with IRF9 for the expression of IFN- $\alpha$ - and IFN- $\beta$-associated genes (reviewed 


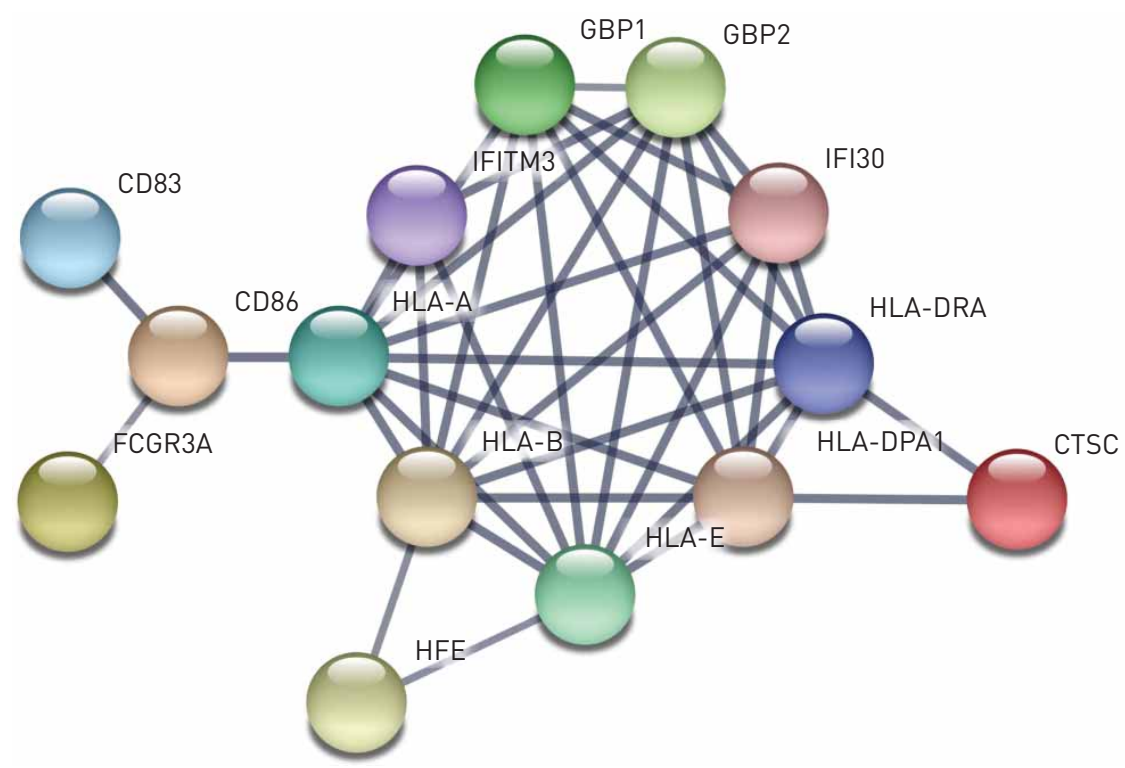

FIGURE 3 STRING network of differentially expressed genes in sputum. Connections reflect protein interactions (either physical or functional).

in [35]). Consequently, interferon responses appear to be suppressed at multiple stages following azithromycin treatment.

As interferon pathways are involved in the induction of inflammatory responses, azithromycin may reduce exacerbation frequency by limiting the inflammation associated with otherwise benign viral infections. In addition, as interferons induce MHC-I and -II expression, suppression of interferon pathways may also explain the observed downregulation of these molecules following azithromycin treatment.

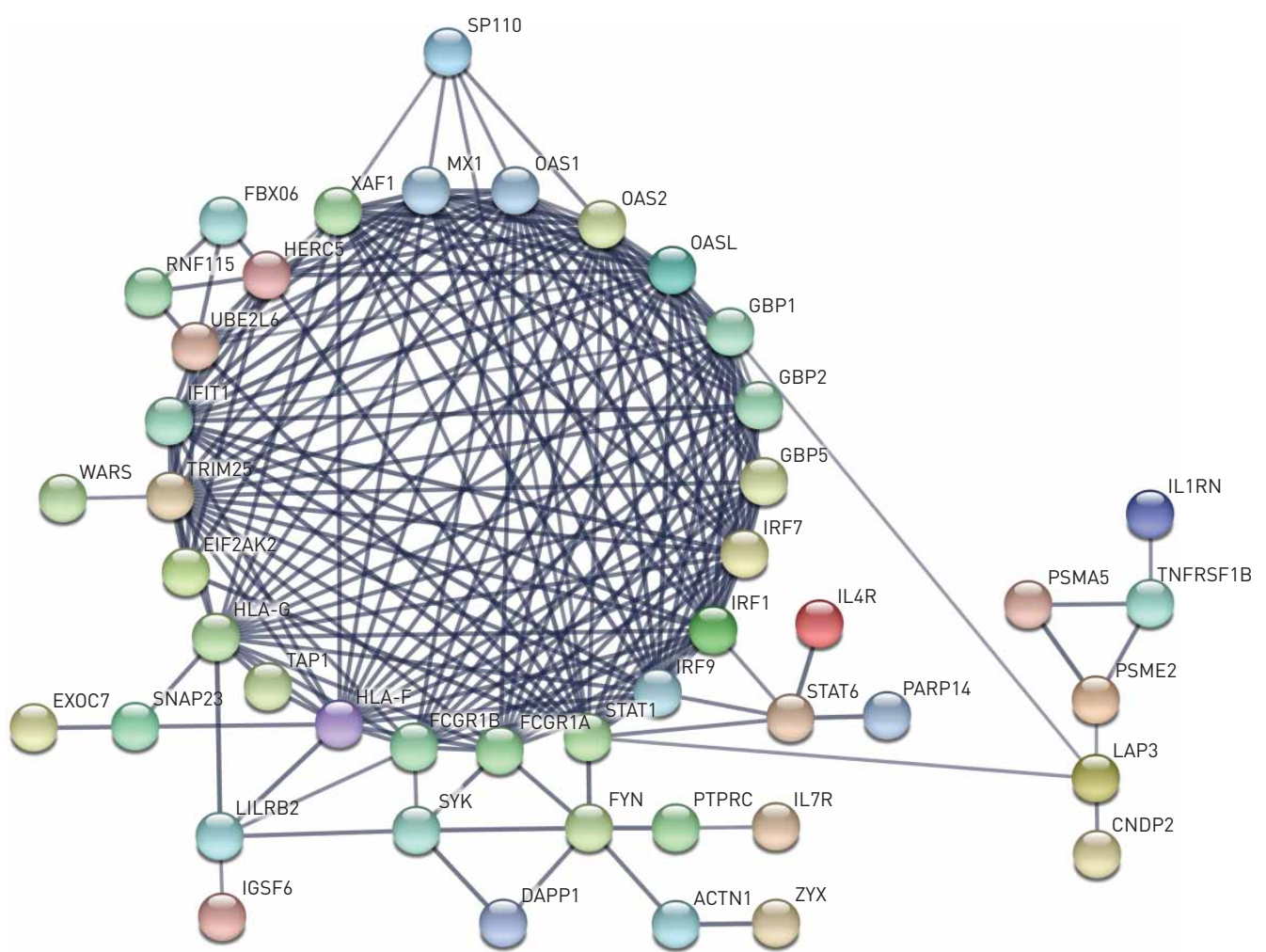

FIGURE 4 STRING network of differentially expressed genes in blood. Connections reflect protein interactions (either physical or functional). 
However, as the induction of interferon responses is typically associated with infection, it is unclear whether these changes are a direct effect of azithromycin (i.e. through modulation of interferon signalling pathways) or secondary to a reduced microbial load from anti-microbial activity. While a trend towards a reduction in bacterial load was observed in these patients, it was not statistically significant [8]. The original study was, however, insufficiently powered to adequately explore this relationship, and so the delineation of azithromycin's immunomodulatory and antimicrobial properties remains unclear.

Previous studies have demonstrated that azithromycin increases viral-induced interferon responses [25, 28, 29]. In the absence of acute infection, however, azithromycin did not affect interferon expression [25, 28, 29], leading PORTER et al. [28] to suggest that macrolides such as azithromycin may work by "priming" interferon pathways. Our results indicate that in the absence of acute viral infection, azithromycin acts as a suppressor of interferon and other inflammatory pathways. Alternatively, a long-term reduction in viral loads by azithromycin may also explain these results. While azithromycin treatment has been shown to

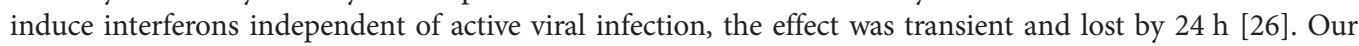
results, taken after 12 weeks of azithromycin treatment, support the notion that this effect is not sustained. Consequently, it is unlikely that infection-independent induction of interferons plays a role in the efficacy of long-term azithromycin treatment.

In the airways, azithromycin was associated with the downregulation of the surface receptor CD86, as well as the cytokines CCL5, CCL23 and CKLF. Through its interaction with CD28, CD86 (B7-2) is a co-stimulatory molecule required for the activation and continued survival of T-cells. CCL5 (RANTES) [36], CCL23 (MPIF-1) [37] and CKLF [38] are chemokines involved in the chemotaxis of numerous leukocytes including T-cells, neutrophils and monocytes. These results support previous studies that have shown that azithromycin supresses T-cell activation [31] and suggest that it may also limit T-cell migration.

Azithromycin was also associated with the downregulation of several other genes involved in immune signalling, including TNFRSF1B (tumour necrosis factor- $\alpha$ receptor), FCGR1a and FCGR1b (constituents of the IgG receptor), ILF2 (transcription factor required for the expression of interleukin (IL)-2 by T-cells) and SYK (a tyrosine kinase involved in B-cell receptor, T-cell receptor and Fc receptor signalling). Most striking, however, were the effects of azithromycin on the IL-4 signalling pathway, which is critical for T-helper (Th)2 differentiation. The IL-4 receptor (IL4R) was downregulated, as were STAT6, a transcription factor activated by the IL-4 receptor that forms STAT6-STAT6 homodimers to induce the expression of IL-4 stimulated genes, and PARP14, a polymerase that promotes STAT6-dependent transcription [39, 40]. IRF1, a key transcription factor for Th1 differentiation, was also downregulated. These results further support the notion that azithromycin suppresses T-cell responses.

In this study, azithromycin treatment resulted in larger fold-changes in gene expression in sputum than in blood. This is probably due to the high cellular penetration of azithromycin, which can result in tissue concentrations 10-fold higher than plasma concentrations [21]. These differences are also expected due to the nature of sputum. As the cells of the airways are exposed to more foreign stimulants than those in blood, the expression of inflammatory genes should be higher in general. Consequently, when baseline gene expression is higher, there is a greater capacity for a reduction in gene expression (and therefore a larger fold-change).

Limitations of this study include the use of mixed cell populations in sputum and blood, which reduced the ability to pinpoint a possible mechanism of azithromycin, and a small sample size, which underpowered the study in general. Furthermore, as all patients had neutrophilic COPD, it is not possible to extrapolate our findings to other respiratory diseases. However, the modulation of universal inflammatory pathways, such as those seen in this study, may explain why azithromycin demonstrates such broad efficacy across a range of respiratory diseases.

In summary, this study indicates that long-term, low-dose azithromycin treatment is associated with the downregulation of genes involved in antigen presentation, interferon and T-cell responses, and numerous inflammatory pathways. These changes may contribute to the capacity of azithromycin to reduce exacerbation frequency in COPD, and if so, suggest that it achieves this clinical outcome through broad modulation of the immune system. This study improves our understanding of azithromycin as an immunomodulatory agent and provides potential novel targets for future drug development. Further research is required to determine how azithromycin induces these changes, and whether they can be achieved without the associated risks of long-term antibiotic use.

Acknowledgements: The authors acknowledge the technical assistance of Hayley Candler, Kevin Oreo, Naomi Fibbens, Kellie Fakes, Bridgette Ridewood, Michelle Gleeson, Rebecca Oldham and Natasha Krowchuk (Priority Research Centre for Healthy Lungs and Hunter Medical Research Institute, Faculty of Health and Medicine, The University of Newcastle, Callaghan, Australia). 
Conflict of interest: K.J. Baines has nothing to disclose. T.K. Wright has nothing to disclose. P.G. Gibson reports grants from NHMRC and personal fees from AstraZeneca, GlaxoSmithKline and Novartis, outside the submitted work. H. Powell has nothing to disclose. P.M. Hansbro has nothing to disclose. J.L. Simpson reports grants from NHMRC during the conduct of the study.

Support statement: This project was supported by an NHMRC project grant.

\section{References}

1 Mathers CD, Loncar D. Projections of global mortality and burden of disease from 2002 to 2030. PLoS Med 2006 3: e442.

2 Murray CJ, Vos T, Lozano R, et al. Disability-adjusted life years (DALYs) for 291 diseases and injuries in 21 regions, 1990-2010: a systematic analysis for the Global Burden of Disease Study 2010. Lancet 2012; 380 $2197-2223$.

3 Donaldson GC, Seemungal TA, Bhowmik A, et al. Relationship between exacerbation frequency and lung function decline in chronic obstructive pulmonary disease. Thorax 2002; 57: 847-852.

4 Seemungal TA, Donaldson GC, Paul EA, et al. Effect of exacerbation on quality of life in patients with chronic obstructive pulmonary disease. Am J Respir Crit Care Med 1998; 157: 1418-1422.

5 Soler-Cataluna JJ, Martinez-Garcia MA, Roman Sanchez P, et al. Severe acute exacerbations and mortality in patients with chronic obstructive pulmonary disease. Thorax 2005; 60: 925-931.

6 Gibson PG, Yang IA, Upham JW, et al. Effect of azithromycin on asthma exacerbations and quality of life in adults with persistent uncontrolled asthma (AMAZES): a randomised, double-blind, placebo-controlled trial. Lancet 2017; 390: 659-668.

7 Albert RK, Connett J, Bailey WC, et al. Azithromycin for prevention of exacerbations of COPD. N Engl J Med 2011; 365: 689-698.

8 Simpson JL, Powell H, Baines KJ, et al. The effect of azithromycin in adults with stable neutrophilic COPD: a double blind randomised, placebo controlled trial. PLoS One 2014; 9: e105609.

9 Uzun S, Djamin RS, Kluytmans JA, et al. Azithromycin maintenance treatment in patients with frequent exacerbations of chronic obstructive pulmonary disease (COLUMBUS): a randomised, double-blind, placebo-controlled trial. Lancet Respir Med 2014; 2: 361-368.

10 Saiman L, Marshall BC, Mayer-Hamblett N, et al. Azithromycin in patients with cystic fibrosis chronically infected with Pseudomonas aeruginosa: a randomized controlled trial. JAMA 2003; 290: 1749-1756.

11 Wolter J, Seeney S, Bell S, et al. Effect of long term treatment with azithromycin on disease parameters in cystic fibrosis: a randomised trial. Thorax 2002; 57: 212-216.

12 Altenburg J, de Graaff CS, Stienstra Y, et al. Effect of azithromycin maintenance treatment on infectious exacerbations among patients with non-cystic fibrosis bronchiectasis: the BAT randomized controlled trial. JAMA 2013; 309: 1251-1259.

13 Wong C, Jayaram L, Karalus $\mathrm{N}$, et al. Azithromycin for prevention of exacerbations in non-cystic fibrosis bronchiectasis (EMBRACE): a randomised, double-blind, placebo-controlled trial. Lancet 2012; 380: 660-667.

14 Hui D, Yan F, Chen RH. The effects of azithromycin on patients with diffuse panbronchiolitis: a retrospective study of 29 cases. J Thorac Dis 2013; 5: 613-617.

15 Corris PA, Ryan VA, Small T, et al. A randomised controlled trial of azithromycin therapy in bronchiolitis obliterans syndrome (BOS) post lung transplantation. Thorax 2015; 70: 442-450.

16 Brill SE, Law M, El-Emir E, et al. Effects of different antibiotic classes on airway bacteria in stable COPD using culture and molecular techniques: a randomised controlled trial. Thorax 2015; 70: 930-938.

17 Jones PW, Quirk FH, Baveystock CM, et al. A self-complete measure of health status for chronic airflow limitation. The St. George's Respiratory Questionnaire. Am Rev Respir Dis 1992; 145: 1321-1327.

18 van der Molen T, Willemse BW, Schokker S, et al. Development, validity and responsiveness of the Clinical COPD Questionnaire. Health Qual Life Outcomes 2003; 1: 13.

19 Gibson PG, Wlodarczyk JW, Hensley MJ, et al. Epidemiological association of airway inflammation with asthma symptoms and airway hyperresponsiveness in childhood. Am J Respir Crit Care Med 1998; 158: 36-41.

20 Edgar R, Domrachev M, Lash AE. Gene Expression Omnibus: NCBI gene expression and hybridization array data repository. Nucleic Acids Res 2002; 30: 207-210.

21 Rodvold KA, George JM, Yoo L. Penetration of anti-infective agents into pulmonary epithelial lining fluid: focus on antibacterial agents. Clin Pharmacokinet 2011; 50: 637-664

22 Hodge S, Hodge G, Jersmann $\mathrm{H}$, et al. Azithromycin improves macrophage phagocytic function and expression of mannose receptor in chronic obstructive pulmonary disease. Am J Respir Crit Care Med 2008; 178: 139-148.

23 Hodge S, Reynolds PN. Low-dose azithromycin improves phagocytosis of bacteria by both alveolar and monocyte-derived macrophages in chronic obstructive pulmonary disease subjects. Respirology 2012; 17: 802-807.

24 Sugiyama K, Shirai R, Mukae $\mathrm{H}$, et al. Differing effects of clarithromycin and azithromycin on cytokine production by murine dendritic cells. Clin Exp Immunol 2007; 147: 540-546.

25 Gielen V, Johnston SL, Edwards MR. Azithromycin induces anti-viral responses in bronchial epithelial cells. Eur Respir J 2010; 36: 646-654.

26 Menzel M, Akbarshahi H, Bjermer L, et al. Azithromycin induces anti-viral effects in cultured bronchial epithelial cells from COPD patients. Sci Rep 2016; 6: 28698.

27 Menzel M, Akbarshahi H, Tufvesson E, et al. Azithromycin augments rhinovirus-induced IFN $\beta$ via cytosolic MDA5 in experimental models of asthma exacerbation. Oncotarget 2017; 8: 31601-31611.

28 Porter JD, Watson J, Roberts LR, et al. Identification of novel macrolides with antibacterial, anti-inflammatory and type I and III IFN-augmenting activity in airway epithelium. J Antimicrob Chemother 2016; 71: 2767-2781

29 Schogler A, Kopf BS, Edwards MR, et al. Novel antiviral properties of azithromycin in cystic fibrosis airway epithelial cells. Eur Respir J 2015; 45: 428-439.

30 Hodge S, Hodge G, Holmes M, et al. Increased CD8 T-cell granzyme B in COPD is suppressed by treatment with low-dose azithromycin. Respirology 2015; 20: 95-100.

31 Ratzinger F, Haslacher H, Poeppl W, et al. Azithromycin suppresses $\mathrm{CD} 4^{+} \mathrm{T}$-cell activation by direct modulation of mTOR activity. Sci Rep 2014; 4: 7438. 
32 Kanoh S, Rubin BK. Mechanisms of action and clinical application of macrolides as immunomodulatory medications. Clin Microbiol Rev 2010; 23: 590-615.

33 Blum JS, Wearsch PA, Cresswell P. Pathways of antigen processing. Annu Rev Immunol 2013; 31: 443-473.

34 Van Kaer L, Ashton-Rickardt PG, Ploegh HL, et al. TAP1 mutant mice are deficient in antigen presentation, surface class I molecules, and CD4-8 ${ }^{+} \mathrm{T}$ cells. Cell 1992; 71: 1205-1214.

35 Villarino AV, Kanno Y, O’Shea JJ. Mechanisms and consequences of Jak-STAT signaling in the immune system. Nat Immunol 2017; 18: 374-384.

36 Schall TJ, Bacon K, Toy KJ, et al. Selective attraction of monocytes and T lymphocytes of the memory phenotype by cytokine RANTES. Nature 1990; 347: 669-671.

37 Patel VP, Kreider BL, Li Y, et al. Molecular and functional characterization of two novel human C-C chemokines as inhibitors of two distinct classes of myeloid progenitors. J Exp Med 1997; 185: 1163-1172.

38 Han W, Lou Y, Tang J, et al. Molecular cloning and characterization of chemokine-like factor 1 (CKLF1), a novel human cytokine with unique structure and potential chemotactic activity. Biochem J 2001; 357: 127-135.

39 Mehrotra P, Riley JP, Patel R, et al. PARP-14 functions as a transcriptional switch for Stat6-dependent gene activation. J Biol Chem 2011; 286: 1767-1776.

40 Takeda K, Tanaka T, Shi W, et al. Essential role of Stat6 in IL-4 signalling. Nature 1996; 380: 627-630. 